\title{
Quantifying the defect-dominated size effect of fracture strain in single crystalline $\mathrm{ZnO}$ nanowires
}

\author{
Mo-Rigen He, ${ }^{1}$ Pan Xiao, ${ }^{2}$ Jiong Zhao, ${ }^{1}$ Sheng Dai, ${ }^{1}$ Fujiu Ke, ${ }^{2,3}$ and Jing Zhu ${ }^{1, a)}$ \\ ${ }^{1}$ Beijing National Center for Electron Microscopy, Laboratory of Advanced Materials, Department of \\ Materials Science and Engineering, Tsinghua University, Beijing 100084, People's Republic of China \\ ${ }^{2}$ The State Key Laboratory of Nonlinear Mechanics, Institute of Mechanics, CAS, Beijing 100190, \\ People's Republic of China \\ ${ }^{3}$ School of Physics, Beihang University, Beijing 100191, People's Republic of China
}

(Received 29 March 2011; accepted 16 April 2011; published online 20 June 2011)

\begin{abstract}
The diameter (D) dependence of fracture strains in [0001]-oriented single crystalline $\mathrm{ZnO}$ nanowires (NWs) with $\mathrm{D}$ ranging from 18 to $114 \mathrm{~nm}$ is experimentally revealed via in situ uniaxial tension and is well understood based on an analytical model developed by combining molecular dynamics simulations with fracture mechanics theories. We show that the scattered fracture strains are dominated by the effective quantities of atomic vacancies, and their lower bound follows a power-form scaling law, resembling the Griffith-type behavior of single critical defects with diameter-dependent sizes, when $\mathrm{D}$ is larger than a critical $\mathrm{D}_{\mathrm{C}}$. In addition, theoretical strength is expected in NWs with $\mathrm{D}<\mathrm{D}_{\mathrm{C}}$. Our studies provide a simple, but basic, understanding for the size effect of strengths in single crystalline NWs. (C) 2011 American Institute of Physics. [doi:10.1063/1.3594655]
\end{abstract}

\section{INTRODUCTION}

Strength is a basic mechanical property for both structural and functional materials. In macroscopic specimens, the fracture strength $\left(\sigma_{\mathrm{FS}}\right)$ is typically lower than $0.1 \%$ of the Young's modulus (YM) and is dominated by a large amount of critical defects, ${ }^{1}$ the size distribution of which leads to the intrinsic scattering of $\sigma_{\mathrm{FS}}$. As depicted by the Weibull statistics, ${ }^{2}$ the expected $\sigma_{\mathrm{FS}}$ of specimens increase as their characteristic sizes decrease, a condition known as size effect, which has been the core issue of fracture mechanics since Griffith. ${ }^{1}$

Recently, increasing attention paid to the mechanical properties in nanowires (NWs) ${ }^{3-8}$ and nanotubes (NTs) ${ }^{9-11}$ is extending the size effect into the nanoscale. Although the defect-dominated $\sigma_{\mathrm{FS}}$ in NTs have been well understood based on Griffith's theory ${ }^{10}$ and atomistic simulations, ${ }^{11}$ for NWs, there were merely some preliminary experimental results on the size effect of $\sigma_{\mathrm{FS}}$. For instance, Wen et al. reported a linear relationship between $\sigma_{\mathrm{FS}}$ and diameter (D) in $\mathrm{ZnO} N W s,{ }^{4}$ Zhu et al. suggested that the $\sigma_{\mathrm{FS}}$ in Si NWs depended log-linearly on the surface areas, ${ }^{8}$ and Agrawal et al. attributed the $\sigma_{\mathrm{FS}}$ in $\mathrm{ZnO} \mathrm{NWs}$-which slightly decreased with increasing surface area-to the defects near the NW surfaces, based on Weibull statistical analysis and molecular dynamics (MD) simulations. ${ }^{5}$ However, the quantitative mechanisms for the size effect (i.e., how the scattered $\sigma_{\mathrm{FS}}$ are related to the NW diameters and, especially, how they are determined by the real microstructural defects in NWs) have not been theoretically clarified yet, but they are of basic importance for the potential application of NWs in

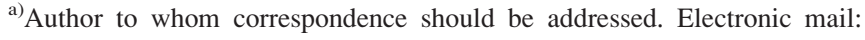
jzhu@mail.tsinghua.edu.cn.
}

nanoelectromechanical devices with predictable and reproducible responses. ${ }^{12}$

In our previous experiments, ${ }^{13}$ methodologies were developed for in situ uniaxial tensile testing using a scanning electron microscope (SEM). The diameter dependence of $\sigma_{\mathrm{FS}}$ has also been revealed in a concise form. ${ }^{14}$ Herein, we report detailed theoretical studies on the size effect of fracture strains $\left(\varepsilon_{\mathrm{FS}}\right)$, which is related to the quantities of native point defects as well as to the critical defect sizes in Griffith's theory. An analytical scaling law is finally derived.

\section{RESULTS AND DISCUSSIONS}

Similar to $\sigma_{\mathrm{FS}}, \varepsilon_{\mathrm{FS}}$ in single crystalline $\mathrm{ZnO}$ NWs with D ranging from 18 to $114 \mathrm{~nm}$ were measured via in situ SEM. The experimental details can be found elsewhere; ${ }^{13}$ in short, each individual NW was axially aligned with the tensile direction and was loaded by deflecting a cantilever [see Fig. 1(a)]. Based on a series of SEM images, the stress-strain $(\sigma-\varepsilon)$ curve was calculated [see Fig. 1(b)].

The diameter dependence of $\varepsilon_{\mathrm{FS}}$ was then revealed [Fig. $1(\mathrm{c})]$. We hereinafter focused on $\varepsilon_{\mathrm{FS}}$ instead of on the conventionally discussed $\sigma_{\mathrm{FS}}$, seeing that the measurement error in the strain is smaller than that in the stress; ${ }^{15}$ moreover, the ideal strain $\left(\varepsilon_{\mathrm{th}}\right.$, i.e., the $\varepsilon_{\mathrm{FS}}$ in defect-free $\left.\mathrm{ZnO} \mathrm{NWs}\right)$ is more insensitive to $\mathrm{D}$ than is the ideal strength $\left(\sigma_{\mathrm{th}}\right)$, which means that the size effect of $\sigma_{\mathrm{FS}}$ is partly contributed by the surface-dominated diameter dependence of $\mathrm{YM}^{13}{ }^{13}$ whereas that of $\varepsilon_{\mathrm{FS}}$ is mostly defect-dominated. ${ }^{15}$ The remarkable scattering of $\varepsilon_{\mathrm{FS}}$ can thus be attributed to the size distribution of critical defects, and their size effect results from the reduction of critical defects with decreasing $\mathrm{D}$, which is in accordance with previous qualitative reports. ${ }^{3-8}$

Concerning critical defects, Weibull statistical analysis is promising for revealing their size and spatial distribution 

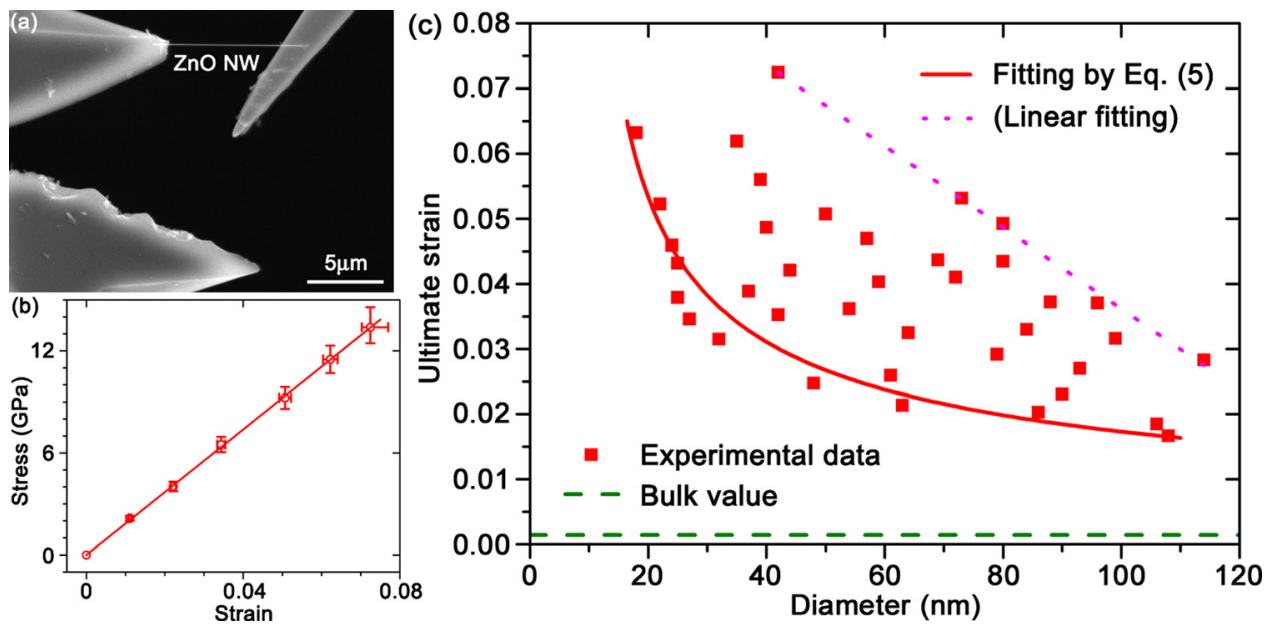

FIG. 1. (Color online) (a) A typical SEM image of the in situ uniaxial tensile testing of a $\mathrm{ZnO} \mathrm{NW}$ and (b) the stressstrain $(\sigma-\varepsilon)$ curve. (c) The experimental diameter dependence of $\varepsilon_{\mathrm{FS}}$ (solid squares). The solid curve fits the lower bound of $\varepsilon_{\mathrm{FS}}$ using a power-form scaling law, and the dotted line is a phenomenological linear fitting of the upper bound. The dashed line shows the typical $\varepsilon_{\mathrm{FS}}$ in bulk $\mathrm{ZnO}(<0.1 \%)$. in specimens. ${ }^{2}$ For NWs, it was conventionally assumed that for a mass of critical defects uniformly distributed on side surfaces, the probability of fracture $\left(\mathrm{P}_{\mathrm{f}}\right)$ under a strain $\varepsilon$ was related to the dimensionless surface area $(\mathrm{S}):^{5}$

$$
P_{f}\left(\varepsilon_{F S}<\varepsilon\right)=1-\exp \left[-S\left(\frac{\varepsilon}{\varepsilon_{0}}\right)^{m}\right] .
$$

Here, $\varepsilon_{0}$ and $m$ depict the expected value and dispersion of $\varepsilon_{\mathrm{FS}}$, respectively, for specimens with a unit S. Alternatively, a modified statistics was recently proposed for NTs having few (e.g., only one) critical defects, ${ }^{16,17}$ where $\mathrm{P}_{\mathrm{f}}$ and the expected strength $\varepsilon_{0}$ were no longer related to specimen sizes:

$$
P_{f}\left(\varepsilon_{F S}<\varepsilon\right)=1-\exp \left[-\left(\frac{\varepsilon}{\varepsilon_{0}}\right)^{m}\right]
$$

As can be seen in Fig. 2, our experimental data were fitted unexpectedly well using Eq. (1b), yielding a correlation factor $\left(\mathrm{R}^{2}\right)$ close to 1 , as were the values of $\varepsilon_{\mathrm{FS}}$ in $\mathrm{ZnO} N W \mathrm{~s}$ measured via in situ free-end bending. ${ }^{3}$ In addition, the transverse intercept of the fitting curve was larger for bending $\left(\varepsilon_{0}=0.058\right)$ than for tension $\left(\varepsilon_{0}=0.043\right)$, manifesting the effect of the loading mode that has been well understood in bulk specimens. ${ }^{2}$ In contrast, the conventional size-dependent statistics [Eq. (1a)] cannot work so satisfactorily. Therefore, the real microstructural defects in the tested NWs should behave as single critical defects, and the effect of surface defects can be ruled out. This knowledge from Weibull statistical analysis also agrees well with the key assumption that our tested $\mathrm{ZnO}$ NWs are free of planar defects and evident surface flaws. ${ }^{14}$

On the other hand, our recent in situ cathodoluminescence experiments convincingly related the size effect of strength with the quantities of native point defects in NWs, ${ }^{14}$ which seems counterintuitive seeing that a single atomic vacancy, even located on the NW surfaces, cannot degrade $\varepsilon_{\mathrm{FS}}$ to less than $80 \%$ of $\varepsilon_{\mathrm{th}}$, ${ }^{10}$ while such a value is still far larger than most of our experimental data. Hence, MD simulations were carried out, aimed at finding the "combination effects" of discrete point defects.

The uniaxial tensile loading of bulk $\mathrm{ZnO}$ and [0001]-oriented NWs were modeled using the Large-scale Atomic/Molecular Massively Parallel Simulator. ${ }^{18,19}$ The supercell for pristine bulk $\mathrm{ZnO}$ was generated by repeating the wurtzite unit cell [see Fig. 3(a)] by 4, 6, and 12 units along the [2 $1 \overline{1} 0$ ], [0110], and [0001] axes, respectively, and periodic boundary conditions were applied in these three directions. The supercell for a NW with $\mathrm{D}=3.6 \mathrm{~nm}$ and a length of $9.4 \mathrm{~nm}$ is shown in Fig. 3(a). The short-range atomic interactions were
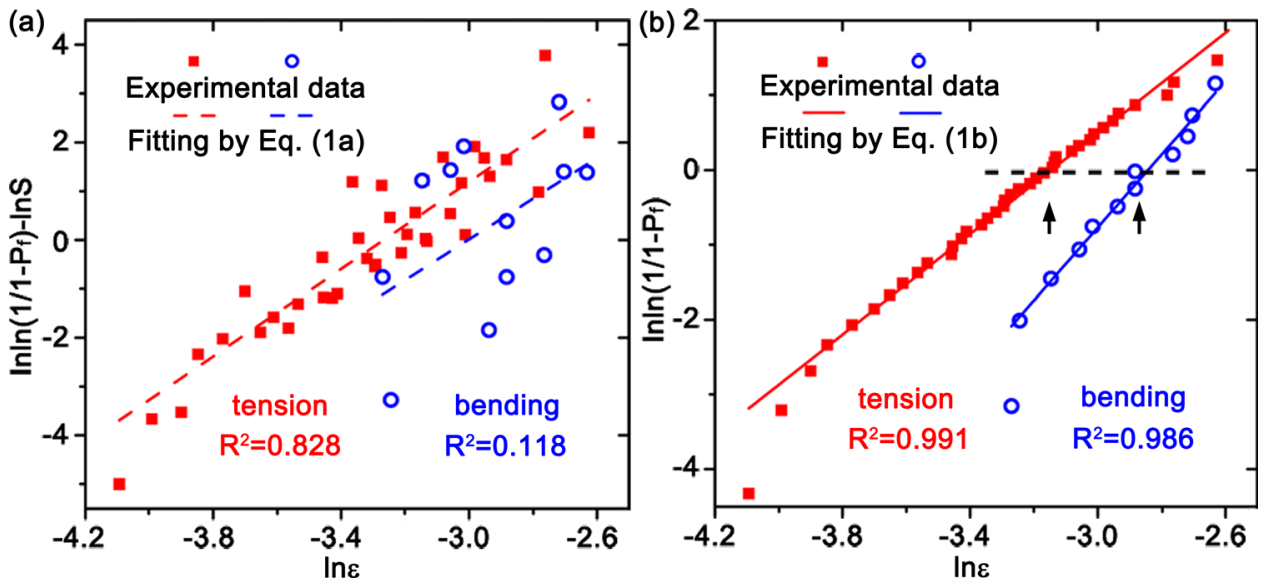

FIG. 2. (Color online) Weibull statistics of the $\varepsilon_{\mathrm{FS}}$ measured by uniaxial tension (solid squares; data from this work) and free-end bending (open circles; data reused with permission from $\mathrm{C}$. Q. Chen and J. Zhu, Appl. Phys. Lett. 90, 043105 (2007). Copyright 2007, American Institute of Physics). The total $\mathrm{N} \varepsilon_{\mathrm{FS}}$ were ranked in ascending order, and $\mathrm{P}_{\mathrm{f}}$ was defined as $(\mathrm{i}-0.5) / \mathrm{N}$ corresponding to the ith $\varepsilon_{\mathrm{FS}}$. The data point $\mathrm{i}=1$ is not used in linear-fitting (Ref. 2). (a) Fitting using Eq. (1a). (b) Fitting using Eq. (1b). The arrows indicate the value of $\ln \varepsilon_{0}$. 

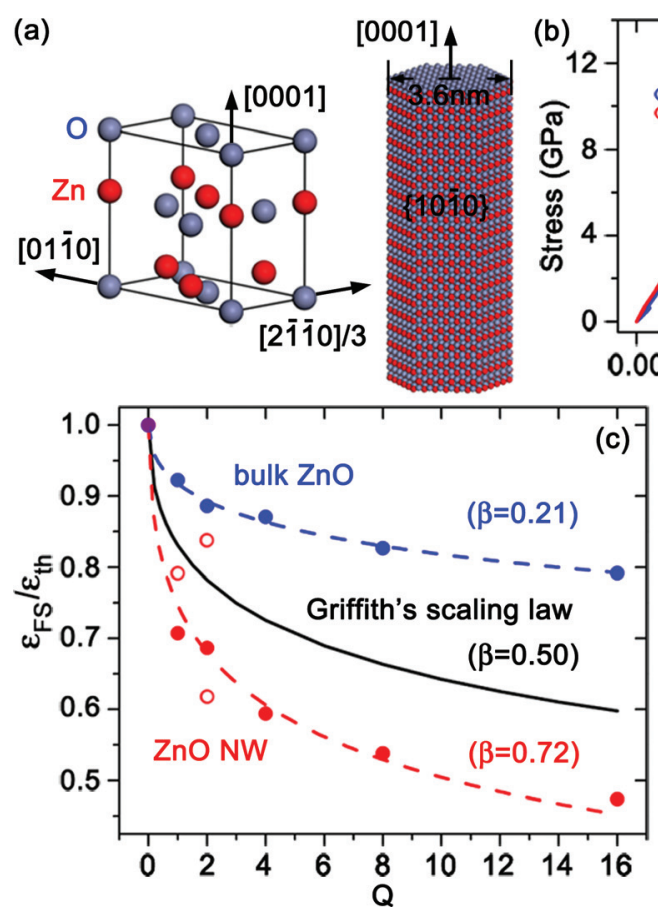
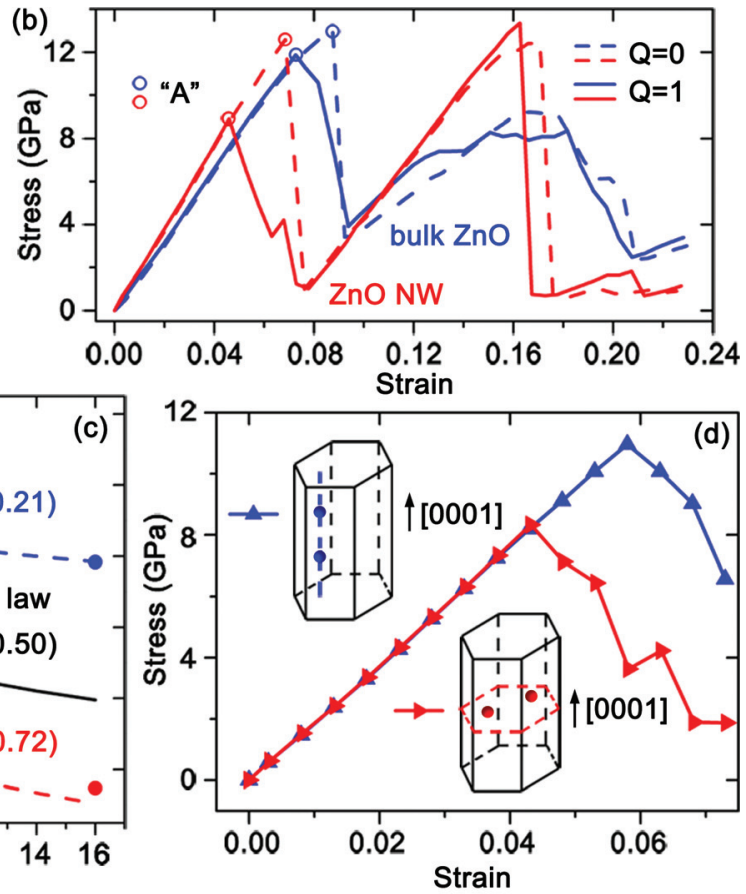

FIG. 3. (Color) (a) The wurtzite unit cell of $\mathrm{ZnO}$ and a supercell of the simulated NW with $\{10 \overline{1} 0\}$ side surfaces and a periodic boundary condition applied on the [0001] axis. (b) Calculated $\sigma-\varepsilon$ curves of bulk $\mathrm{ZnO}$ (blue) and NWs (red). Dashed lines: defect-free; solid lines: one VP randomly introduced. (c) Dependence of $\varepsilon_{\mathrm{FS}}$ (normalized by $\varepsilon_{\mathrm{th}}$ ) on the quantity (Q) of VPs in bulk ZnO (blue) and NWs (red). For the randomly introduced VPs, the MD-simulated results (solid circles) are fitted by Eq. (3) (dashed lines), and the solid line shows classic theory with $\beta=0.50$. The open circle at $\mathrm{Q}=1$ corresponds to another random VP, and those at $\mathrm{Q}=2$ correspond to special configurations of VPs, as shown in (d). (d) The $\sigma-\varepsilon$ curves of the NW containing two VPs (indicated with colored circles) that are aligned parallel to the [0001] axis (blue) and the (0001) plane (red). described by a Buckingham-type potential, ${ }^{20}$ and the Coulomb interactions were calculated via Wolf summation. ${ }^{21}$ The initial relaxation, quasistatic loading, and calculation of $\sigma-\varepsilon$ curves were then performed according to our previous work. $^{22}$ As Fig. 3(b) shows, a phase transformation from wurtzite to a body-centered-tetragonal lattice is predicted as before at points "A". 5,22 However, such post-elastic behaviors have actually not been observed yet during the in situ tensile testing of individual $\mathrm{ZnO}$ NWs, probably because that displacement-controlled tensile loading has not been realized in the nanoscale. As a result, the stress drop accompanied by the phase transformation can immediately fracture ZnO NWs that are intrinsically brittle materials. ${ }^{5} \mathrm{We}$ also noted a recent first-principle calculation that suggested that the phase transformation can not happen until the pristine NW fractured with an $\varepsilon_{\text {th }}$ as high as $20 \% .{ }^{23}$ Concerning the effect of defects on strength, however, no difference has been provided in comparison to MD simulations. Overall, it is yet reasonable to define the $\varepsilon_{\mathrm{FS}}$, in our current MD studies, based on points "A".

Then, the effect of point defects on $\varepsilon_{\mathrm{FS}}$ was simulated by intentionally introducing vacancy-pairs (VPs) one by one into the supercell. Each time, a random $\mathrm{O}^{2-}$ site and one of its nearest-neighboring $\mathrm{Zn}^{2+}$ site were simultaneously replaced by vacancies, in order to preserve the charge equilibrium. For the randomly introduced VPs, Fig. 3(c) reveals that $\varepsilon_{\mathrm{FS}}$ (in both the bulk and the NWs) decreased as the quantity (Q) of VPs increased. Moreover, a range of $\varepsilon_{\mathrm{FS}}$ can be obtained for a given $\mathrm{Q}$ (take $\mathrm{Q}=1$ and 2, for example). As revealed in Fig. 3(d), $\varepsilon_{\mathrm{FS}}$ can be degraded more remarkably when the two VPs are aligned parallel to the (0001) cleavage plane, which does suggest some interactions between discrete point defects, such as stress concentrations and defect aggregations via atomic diffusions. ${ }^{24}$
After all, $\varepsilon_{\mathrm{FS}}$ not only depended on the quantity of point defects, but also were affected by the spatial configuration of these VPs; we can thus define the effective sizes (n) of the single critical defects, which uniquely determine $\varepsilon_{\mathrm{FS}}$, as

$$
n^{2}=\eta Q
$$

Here, $\mathrm{n}$ is normalized by the in-plane lattice constant. For each specific NW with a total of Q VPs, $\eta$ depicts the proportion of the VPs involved in the above-suggested interactions, as well as the overall effects of the spatial configurations of these "active" defects. As indicated by Fig. 3(d), the upper bound of $\mathrm{n}$ ( $\operatorname{simply}$ let $\eta=1$ ) corresponds to a sharp planar crack perpendicular to the [0001] NW axis, the fracture mechanics model for which can be analytically derived as

$$
\varepsilon_{F S}(n)=\varepsilon_{t h}(1+n)^{-\beta}
$$

based on an atomic-scale, i.e., "quantized" Griffith's theory that has been widely applied to the $\sigma_{\mathrm{FS}}$ in NTs. ${ }^{10}$ Nonetheless, Eq. (3) should generally work for $n$, because the geometric effect of the crack-tip radius ${ }^{10}$ can be included in the factor $\eta$.

First, Eq. (2) was confirmed by fitting the MD-simulated $\varepsilon_{\mathrm{FS}}(\mathrm{Q})$ relationships using Eq. (3). A constant $\eta_{\text {average }}$ was supposed to calculate $\mathrm{n}$ for the randomly introduced VPs. As seen in Fig. 3(c), the power factors $\beta=0.21$ and 0.72 were yielded for the bulk and the NW $(\mathrm{D}=3.6 \mathrm{~nm})$, respectively, implying that $\varepsilon_{\mathrm{FS}}$ in NWs is more sensitive to Q than that in the bulk, because the point defects near free surfaces can lead to more intense stress concentrations than those in bulk materials.

The diameter dependence of $n(D)$ in the experimentally tested $\mathrm{ZnO}$ NWs was then determined by applying Eq. (3) to the $\varepsilon_{\mathrm{FS}}(\mathrm{D})$ as measured in Fig. 1(c), where D ranged from 18 to $114 \mathrm{~nm}$. A classic $\beta=0.50$ representing the Griffith's 
scaling law was utilized as a reasonable simplification, and $\varepsilon_{\text {th }}$ was assumed to be the constant $\sim 0.09$ that was simulated in bulk $\mathrm{ZnO} .{ }^{15}$ Straightforwardly, the scattering of experimental $n(D)$ for a given D (see Fig. 4) resulted from the stochastic and specimen-specific factor $\eta$; moreover, the diameterdependent upper bound of $n(D)$ can be simply modeled by assuming $\eta=1$, which means that all of the native point defects in NWs are "active" in dominating their minimum strengths. As a result, one has $\mathrm{n}_{\max }{ }^{2}(\mathrm{D})=\mathrm{Q}(\mathrm{D})=\lambda \mathrm{D}^{2} \mathrm{C}(\mathrm{D})$, where $\lambda$ is the length scale depicting the interactions between point defects, and the concentration of point defects $\mathrm{C}(\mathrm{D})=\mathrm{C}_{0} \exp \left(-\Delta \mathrm{p} \cdot \Omega / \mathrm{k}_{\mathrm{B}} \mathrm{T}\right)$. Here, $\mathrm{C}_{0}$ is the concentration in bulk materials, $\Omega$ is the volume of the $\mathrm{Zn}^{2+}$ and $\mathrm{O}^{2-}$ vacancies, $\mathrm{k}_{\mathrm{B}} \mathrm{T}$ is the Boltzmann factor, and $\Delta \mathrm{p}=2 \tau / \mathrm{D}$ ( $\tau$ is the surface tension in $\{10 \overline{1} 0\}$ side surfaces). ${ }^{14}$ The sizedependent reduction of critical defect sizes thus can be derived as

$$
n_{\max }(D)=\sqrt{\lambda C_{0}} D \exp \left(-\frac{\tau \Omega}{D k_{B} T}\right) \approx \alpha \max \left(D-D_{C}, 0\right),
$$

where $\alpha$ is a constant. Hence, a critical NW diameter was derived as $D_{C}=\tau \Omega / k_{B} T$, concerning the $\mathrm{ZnO}$ NWs in experiments, and Eq. (4) predicted that critical defects no longer existed in NWs with $\mathrm{D}<\mathrm{D}_{\mathrm{C}}$; in other words, the strength became insensitive to defects. Similar behavior has been proposed in nano-laminated composites with $\mathrm{D}_{\mathrm{C}}$ around $100 \mathrm{~nm},{ }^{25}$ but not yet in single crystalline NWs.

As Fig. 4 shows, fitting the experimental upper bound of $\mathrm{n}(\mathrm{D})$ using Eq. (4) yielded a good linear correlation; moreover, extrapolating the fitting curve to $\mathrm{n}_{\max }=0$ yielded $\mathrm{D}_{\mathrm{C}}=11.6 \mathrm{~nm}$, which agreed quantitatively well with $\tau \Omega$ / $\mathrm{k}_{\mathrm{B}} \mathrm{T}=10.8 \mathrm{~nm}$, where the surface tension in $\{10 \overline{1} 0\}$ is estimated as $\tau \sim 3.4 \mathrm{~N} / \mathrm{m},{ }^{26}$ the ionic radii of $\mathrm{O}^{2-}$, and $\mathrm{Zn}^{2+}$ are $1.40 \AA$ and $0.74 \AA$, respectively, and we assume $\mathrm{T}=300 \mathrm{~K}$. Therefore, the Griffith-type fracture mechanics for the effective $n(D)$ of the single critical defects, which is defined as Eq. (2), was experimentally supported.

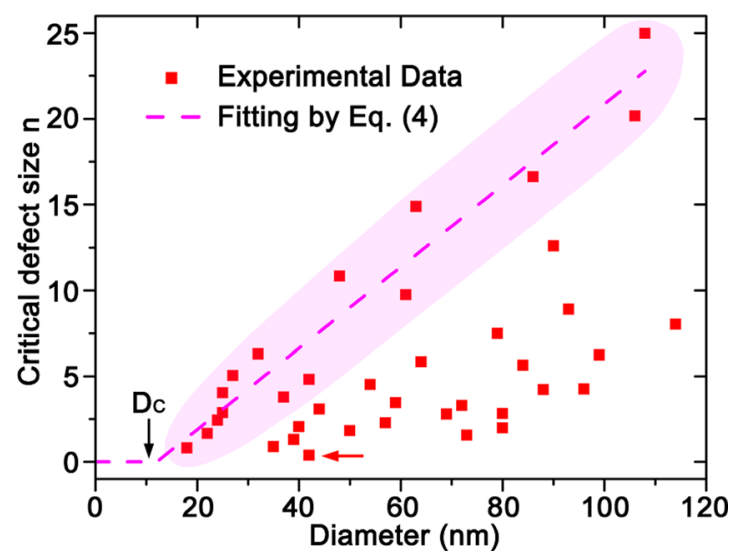

FIG. 4. (Color online) Experimental diameter-dependent n(D) of the effective single critical defect (solid squares). The dashed line is linear fitting of the upper bound of $n(D)$ in the shadowed region, and the transverse extrapolation predicts a critical $\mathrm{D}_{\mathrm{C}}=11.6 \mathrm{~nm}$, below which $\mathrm{n}(\mathrm{D})$ converges to zero. The arrow on the left indicates a NW with n close to one unit cell.
We finally return to the size effect of $\varepsilon_{\mathrm{FS}}$. As seen in Fig. 1, the upper-bound of $\varepsilon_{\mathrm{FS}}$ increased with decreasing D, following a linear relationship that has nonetheless not been quantitatively modeled yet, as further probing into the fracture mechanism is still needed in order to find whether the minimum of the "active" defects $(\eta \mathrm{Q})$ is random or diameter-dependent; while for the lower-bound of $\varepsilon_{\mathrm{FS}}$, the following power law for $\mathrm{D}>\mathrm{D}_{\mathrm{C}}$ can be obtained by combining Eqs. (3) and (4):

$$
\varepsilon_{F S}^{\min }(D)=\varepsilon_{t h}\left[1+\alpha\left(D-D_{C}\right)\right]^{-\beta} . \quad \text { for } \mathrm{D}>\mathrm{D}_{\mathrm{C}}
$$

Fitting the experimental $\varepsilon_{\mathrm{FS}}(\mathrm{D})$ with Eq. (5) yielded $\varepsilon_{\text {th }}=0.11$ and $\beta=0.52$ [see Fig. 1(c)], agreeing qualitatively with Griffith's classic theories where $\varepsilon_{\text {th }} \sim 0.1$ and $\beta \sim 0.5$. $^{1,10}$ Ultimately, a modified power-form scaling law for the size effect of strengths was analytically derived, and we concluded, for the first time, that the classic fracture mechanics theories also work well for single crystalline NWs, as long as the effective quantities of point defects are regarded as critical defects.

\section{CONCLUSIONS}

In summary, the diameter dependence of $\varepsilon_{\mathrm{FS}}$ in single crystalline $\mathrm{ZnO} \mathrm{NWs}$ is experimentally revealed via in situ uniaxial tension and analytically modeled based on fracture mechanics theories and MD simulations. The scattered $\varepsilon_{\mathrm{FS}}$ are dominated by the effective quantities of atomic vacancies, and the lower bound of $\varepsilon_{\mathrm{FS}}$ follows a power law, resembling the Griffith-type behavior of single critical defects with diameter-dependent sizes. In addition, the theoretical strength is predicted in NWs with $\mathrm{D}<\mathrm{D}_{\mathrm{C}}$ (around $12 \mathrm{~nm}$ ). Our studies account for a very simple case of single crystalline NWs; however, this should be the basis for fully understanding the size effect of strengths in the nanoscale.

We still need to state that the detailed mechanisms of fracture remain unclear, since it is challenging yet to (i) directly inspect the atomic-scale behaviors of point defects using in situ TEM and (ii) measure the post-elastic $\sigma-\varepsilon$ responses via displacement-controlled tensile experiments. More attention should be paid to experimental improvements in order to understand and ultimately control the strength properties in nanomaterials.

\section{ACKNOWLEDGMENTS}

Financial support from the National Natural Science Foundation of China $(50831001,10772012)$ and the National 973 Project of China (2009CB623700, 2007CB814803) is acknowledged. The experiments made use of the resources of the Beijing National Center for Electron Microscopy, and the computations were carried out at the CNIC Supercomputing Center at the LSEC of CAS.

\footnotetext{
${ }^{1}$ A. A. Griffith, Philos. Trans. R. Soc. London, Ser. A 221, 163 (1921).

${ }^{2}$ W. Weibull, J. Appl. Mech. 18, 293 (1951).

${ }^{3}$ C. Q. Chen and J. Zhu, Appl. Phys. Lett. 90, 043105 (2007).

${ }^{4}$ B. M. Wen, J. E. Sader, and J. J. Boland, Phys. Rev. Lett. 101, 175502 (2008).

${ }^{5}$ R. Agrawal, B. Peng, and H. D. Espinosa, Nano Lett. 9, 4177 (2009).
} 
${ }^{6}$ F. Xu, Q. Q. Qin, A. Mishra, Y. Gu, and Y. Zhu, Nano Res. 3, 271 (2010). ${ }^{7}$ S. Hoffmann, F. Östlund, J. Michler, H. J. Fan, M. Zacharias, S. H. Christiansen, and C. Ballif, Nanotechnology 18, 205503 (2007).

${ }^{8}$ Y. Zhu, F. Xu, Q. Q. Qin, W. Y. Fung, and W. Lu, Nano Lett. 9, 3934 (2009).

${ }^{9}$ M. F. Yu, O. Lourie, M. J. Dyer, K. Moloni, T. F. Kelly, and R. S. Ruoff, Science 287, 637 (2000).

${ }^{10}$ N. M. Pugno and R. S. Ruoff, Philos. Mag. 84, 2829 (2004).

${ }^{11}$ T. Belytschko, S. P. Xiao, G. C. Schatz, and R. S. Ruoff, Phys. Rev. B 65, 235430 (2002); B. Peng, M. Locascio, P. Zapol, S. Y. Li, S. L. Mielke, G. C. Schatz, and H. D. Espinosa, Nature Nanotech. 3, 626 (2008).

${ }^{12}$ Z. L. Wang and J. H. Song, Science 312, 242 (2006).

${ }^{13}$ M. R. He, Y. Shi, W. Zhou, J. W. Chen, Y. J. Yan, and J. Zhu, Appl. Phys. Lett. 95, 091912 (2009).

${ }^{14}$ M. R. He and J. Zhu, Phys. Rev. B 83, 161302R (2011).

${ }^{15}$ See supplementary material at http://dx.doi.org/10.1063/1.3594655 for (i) an estimation of measurement errors in $\varepsilon \mathrm{FS}$ and $\sigma \mathrm{FS}$ and (ii) discussions on the size effects of $\varepsilon_{\mathrm{th}}$ and $\sigma_{\text {th }}$.

${ }^{16}$ N. M. Pugno and R. S. Ruoff, J. Appl. Phys. 99, 024301 (2006).
${ }^{17}$ I. Kaplan-Ashiri, S. R. Cohen, K. Gartsman, V. Ivanovskaya, T. Heine, G. Seifert, I. Wiesel, H. D. Wagner, and R. Tenne, Proc. Natl. Acad. Sci. U.S.A. 103, 523 (2006)

${ }^{18}$ S. J. Plimpton, J. Comput. Phys. 117, 1 (1995).

${ }^{19}$ See http://lammps.sandia.gov for more information on the code and simulator.

${ }^{20}$ D. J. Binks and R. W. Grimes, J. Am. Ceram. Soc. 76, 2370 (1993).

${ }^{21}$ D. Wolf, P. Keblinski, S. R. Phillpot, and J. Eggebrecht, J. Chem. Phys. 110, 8254 (1999).

${ }^{22}$ J. Wang, A. J. Kulkarni, F. J. Ke, Y. L. Bai, and M. Zhou, Comput. Methods Appl. Mech. Eng. 197, 3182 (2008).

${ }^{23}$ R. Agrawal, J. T. Paci, and H. D. Espinosa, Nano Lett. 10, 3432 (2010).

${ }^{24}$ L. S. Hounsome, R. Jones, P. M. Martineau, M. J. Shaw, P. R. Briddon, S. Öberg, A. T. Blumenau, and N. Fujita, Phys. Status Solidi A 202, 2182 (2005); A. Adnan and C. T. Sun, J. Mech. Phys. Solids 58, 983 (2010).

${ }^{25}$ H. J. Gao, B. H. Ji, I. L. Jäger, E. Arzt, and P. Fratzl, Proc. Natl. Acad. Sci. U.S.A. 100, 5597 (2003).

${ }^{26}$ N. Fujimura, T. Nishihara, S. Goto, J. F. Xu, and T. Ito, J. Cryst. Growth 130, 269 (1993). 